\title{
Automated Test Case Generation ANd OPTIMIZATION: A COMPARATIVE REVIEW
}

\author{
Rajesh Kumar Sahoo ${ }^{1}$, Deeptimanta Ojha ${ }^{2}$, Durga Prasad Mohapatra ${ }^{3}$, Manas \\ Ranjan Patra ${ }^{4}$ \\ ${ }^{1}$ Department of Computer Engineering, A.B.I.T, Cuttack \\ ${ }^{2}$ Department of Computer Engineering, A.B.I.T, Cuttack \\ ${ }^{3}$ Department of Computer Engineering, NIT,Rourkela \\ ${ }^{4}$ Department of Computer Engineering, Berhampur University,Berhampur
}

\begin{abstract}
Software testing is the primary phase, which is performed during software development and it is carried by a sequence of instructions of test inputs followed by expected output. Evolutionary algorithms are most popular in the computational field based on population. The test case generation process is used to identify test cases with resources and also identifies critical domain requirements. The behavior of bees is based on population and evolutionary method. Bee Colony algorithm (BCA) has gained superiority in comparison to other algorithms in the field of computation. The Harmony Search (HS) algorithm is based on the enhancement process of music. When musicians compose the harmony through different possible combinations of the music, at that time the pitches are stored in the harmony memory and the optimization can be done by adjusting the input pitches and generate the perfect harmony. Particle Swarm Optimization (PSO) is an intelligence based meta-heuristic algorithm where each particle can locate their source of food at different position.. In this algorithm, the particles will search for a better food source position in the hope of getting a better result. In this paper, the role of Artificial Bee Colony, particle swarm optimization and harmony search algorithms are analyzed in generating random test data and optimized those test data. Test case generation and optimization through bee colony, PSO and harmony search (HS) algorithms which are applied through a case study, i.e., withdrawal operation in Bank ATM and it is observed that these algorithms are able to generate suitable automated test cases or test data in a client manner. This section further gives the brief details and compares between HS, PSO, and Bee Colony (BC) Optimization methods which are used for test case or test data generation and optimization.
\end{abstract}

\section{KEYWORDS}

Bee Colony algorithm, particle swarm optimization, harmony search algorithm, meta-heuristics, test case generation, test case optimization, test data.

\section{INTRODUCTION}

Generations of test cases are based on the requirements. It completely ignores the aspect of system execution. Apart from this, the test case design from program code may cause difficult to imbrute [8]. Test cases may not expose the missing functionalities. The proposed approach focuses the redundancy, test cases, and test case optimization challenges. It uses HS, PSO and Bee colony optimization algorithm to optimize the random test cases. Moreover, this proposed methods inspired the developers to generate random test cases to improve the design quality of DOI:10.5121/ijcsit.2016.8502 
software. This paper is intended to present the result of the outcome of different evolutionary meta heuristic algorithms like HS, pso and bee colony to find the optimum solutions. These solutions are essential for the software construct. Optimization can be represented through the process to find the best result under the given circumstances which might be used for maximizing or minimizing the local or overall optimum value of a function. The evolutionary algorithms like Harmony search, Particle swarm optimization and Bee colony algorithms are discussed in this paper. Z. W. Geem [11] introduced Harmony search method in 2009.By this technique, a flawless state of harmony is observed through the musical process. It is correspondent to generate the optimum value through the musical optimization process. The improvisation of music is a technique where the musician plays various musical notes with divergent types of musical instruments and generates the best amalgamation of tunes with frequency. D.D.Karaboga[7] introduced Bee colony algorithm in 2005. In Bee colony algorithm (BCA), three types of bees are employed, onlooker and scout bees. Those bees will continuously search for the different food source position as per quality in order to replace the solution with new improved solution. J.Kenedy[15] developed Particle swarm optimization algorithm in 1995.Particles searching their foods through the positions and velocities. The best position and velocity is updated with older one. Modification of velocity helps the particle to move faster for searching foods.

The rest of the paper is organized as follows. Section 2 discusses basics of test data automation, over view of harmony search,basic particle swarm optimization and bee colony algorithm.Section 3 is for literature survey, Section 4 represents the fundamentals of Harmony Search (HS) algorithm,particle swarm aoptimization,bee colony algorithm, proposed systems, and methodology. Section 5 focuses the simulation results, Section 6 represents discussion and future scope and Section 7 concludes the paper.

\section{BASIC CONCEPTS}

\subsection{Automated Test DAta Generation}

Testing is the phenomenon of finding errors after executing the programs. Software testing can be defined by many processes designed sequentially and does not do anything unintended [21].The objective of software testing is to finalize the application software against the user requirements. It must have good test coverage to test the application software and perform as per the specifications. For generating list of coverage's, the test cases should be designed with maximum possibilities of finding various errors or bugs [22]. The test cases should be very effective and is measured through the number of defects or errors reported. Generation of test cases or test data is a method to identify the data set which satisfies the criteria. Most of the researchers are used the heuristics approaches for automated generation of test cases or data. Automated test data generation helps to minimizing the time and cost in developing test cases.

\subsection{OVERVIEW OF HARMONY SEARCH}

The harmony search metaheuristic optimization algorithm is based on the music. The harmony search method is inspired by a musician when he composes the music. Usually harmony consists of different possible amalgamations of music pitches saved in the memory. In this technique first, random solutions are directly stored in the harmony memory based on memory considering rate and pitch adjustment rate, and then pitch adjustment distance will be calculated between different 
selected random solutions. The best solution is stored in the memory of harmony by discarding the worst solution.

\subsection{OVerview Of Particle SWarm Optimization}

The particle swarm optimization (PSO) is an evolutionary algorithm motivated by social behavior of grouped migrating birds. Kennedy and Eberhart introduced PSO in 1995 [15] which can solve the optimization problem. Schooling of fish and the behavior of birds in a group inspired a population based optimization method. In a search space each particle stays at a particular position. The quality of each particle position determines its fitness. Particles have their own independent velocity and speed. The velocity of each particle is dependent on its neighbors which gives the best fitness functional value. Generations of test cases are used to identify test cases with resources and also identify critical domain requirements.

\subsection{OVERVIEW OF BEE COLONY OPTIMIZATION}

Bee Colony algorithm (BCA) is a evolutionary population based method which based on the behaviors of the bees .It is developed by Dervis Karaboga[7] for optimization purpose in 2005. Bee colony method states that the bees are found their food source through their foraging behavior. The aims of honey bees are to establish places of food source with highest nectar amount. This algorithm is very popular in computational field .In this algorithm, the bees will search for a best position of food source in the hope to get better result. . The food source position represents a possible set of solutions and the amount of nectar represent corresponding fitness values or quality of all solutions or the food source.

\section{LITERATURE SURVEY}

Kaur Arvinder et.al[1] proposed how different factors like coordination, synchronization are applied in bee colony optimization technique.Through this optimization techniques different kinds of large and complex programs are optimized. Biswal et.al [2] focused how the test case scenarios are derived from activity diagram which may be converted into control flow graph (CFG ) where each node represents an activity and the edges of the flow graph.Euclidean distance is used by Korel [3] to quantify the distance between two paths of the control flow graph. According to Basturk. B et.al and Dahiya. S et.al [4][6] proposed an approach which aimed to generate the optimal test cases with good path coverage. This paper also explains how the bee agents gather the food source through test cases and identify optimal test cases with respect to fitness functional value. According to Geem et al. [11,12] harmony search a metaheuristic population-based algorithm where multiple harmonies are used in parallel which gives better performance with high efficiency. Geem [12] focused the Pitch Adjustment Rate (PAR) function which is used in simulated annealing and it also increases the robustness of the algorithm. It is highly reliable. Geem et al. [13] described how the parameter like Pitch adjustment rate (PAR) increases in a linear way with the number of generations while the bandwidth (BW) decreases exponentially in Harmony Search algorithm. Manjarres et al. [9] focused described various applications of HS like industry, power systems, construction design, and information technology. Das et al. [10] described the background of the power of Harmony Search (HS) which gives the better solutions. The major drawback is user must specify the minimum and maximum bandwidth values. It is difficult to deduce the program which is dependent. Suresh et 
al. [23] described a genetic algorithm used for generation of test data and it also generates the basic path. In this case the genetic algorithm combines the features of local and global test data optimization. According to this paper sequence diagram is converted into control flow graph and generates the optimal test cases using genetic algorithm. Sthamer [14] explained about the test data generation by using structural test coverage through Genetic Algorithms. Lin and Yeh [16] discussed about the generation of test data through Genetic Algorithms automatically. Wegener et al. [17] described a test environment which is used for test data generation in statement and branch testing automatically. S.K. Swain and D.P. Mohapatra[25] discussed about the number or test paths generated through a model and it also covers the coverage criteria like branch coverage, condition coverage and full test path coverage. According to Kaur et al[18] Hybrid Particle Swarm Optimization (HPSO) algorithm for performing in a search space to get an efficient solution through regression testing. According to Mustafa Servet Kiran and Ahmet Babalik[19] bee colony algorithm (BCA) gives optimum solution for combinatorial optimization problems. This technique acts as a decentralized way and self organized. Pargas et al. [20] focused on the Genetic Algorithm is directed by control-dependence graph program under test for searching test data to satisfy the criteria of all-nodes and all-branches. According to Michael et al. [24] the genetic algorithm (GA) is used for automated test data generation to satisfy decision and condition coverage criterion.

\section{Proposed System}

This paper proposed a methodology for generating test cases for withdrawal system of an ATM machine and test cases are optimized by different evolutionary algorithms like Harmony search algorithm (HSA), Particle swarm optimization algorithm (PSOA) and Bee colony algorithm (BCA).These methods are used for evaluating its efficiency and effectiveness for generating the test cases and for maximizing to achieve the goal.

\subsection{Necessity Of Proposed System}

The proposed system is intended to generate an automatic and optimized test case with existing approaches of Harmony Search, PSOA and Bee Colony Algorithm. Optimized test cases may not be helpful in the testing process. It may be required to differentiate between the various test cases. In case of HSA all the system may be initialized with harmony memories. Each harmony memory maintains its own prevailing location. Harmony Search has a memory that helps to maintain the solutions by harmonies. For PSOA the system may be initialized with particle positions. Each particle maintains its locations as test cases. In Bee colony algorithm (BCA) food source positions may be initialized by the system and each food source maintains its positions. This paper also finds out the effectiveness of the proposed approach through the number of test cases or test data.

\subsubsection{HARMONY SEARCH Algorithm (HSA)}

Harmony search is a meta heuristic population-based optimization technique. Through this technique, the problem is represented through different test cases. The quality of each test case is calculated through the fitness value of the problem. The working principle of harmony search technique is inspired by the musician when he composes the music; a musician usually tries different combinations of music pitches which are stored in the harmony memory. Perfect 
harmony search needs a correspondent for generating the process to find the solution which is optimal. The main steps of a harmony search method are:

- Generate random solutions which will be stored in the Harmony Memory (HM).

- Select a random solution from Harmony Memory based factors that are Harmony Memory Considering Rate (HMCR) and Pitching Adjustment Rate (PAR).

- Apply adjustments pitch distance to the selected random solution.

- Compare the fitness function values of the mutated solution or newly formed solution with the worst solution.

- If better solution is found then the worst solution is substituted with the mutated solution available in the harmony memory otherwise the solution is discarded.

- Remember the solution which is best so far.

In this case, mainly two parameters are used. They are HMCR and PAR. Harmony Memory Considering Rate (HMCR) is described as the probability of selecting a particular component or solution available in HM. Pitching Adjust Rate (PAR) describes the probability distribution of the candidate solution for mutation purpose through HM.

The value of Pitching Adjust Rate (PAR) can be calculated as follows.

$$
\begin{aligned}
\mathrm{PAR}=\left(\mathrm{PAR}_{\max }-\mathrm{PAR}_{\min }\right) /\left(\mathrm{it}_{\text {max }}\right) * \mathrm{it} & \left.+\mathrm{PAR}_{\min } \quad .1\right) \\
\text { Where } & \mathrm{PAR}_{\max } \rightarrow \text { maximum pitch adjusting rate } \\
& \mathrm{PAR}_{\min } \rightarrow \text { minimum pitch adjusting rate } \\
& \mathrm{it}_{\max } \rightarrow \text { given maximum iterations } \\
& \text { it } \rightarrow \text { current iteration number }
\end{aligned}
$$

The bandwidth factor 'bw' can be calculated as.

$$
\mathrm{bw}=\mathrm{bw}_{\max } * \exp (\operatorname{coef} * \mathrm{it})
$$

Here the value of 'coef' is evaluated as follows:

$$
\text { coef }=\log \left(\mathrm{bw}_{\min } / \mathrm{bw}_{\max } / \mathrm{it}_{\max }\right)
$$

Where bw $=$ bandwidth

$$
\begin{aligned}
& \mathrm{bw}_{\min }=\text { minimum bandwidth } \\
& \mathrm{bw}_{\max }=\text { maximum bandwidth }
\end{aligned}
$$

The bandwidth factor bw is used to alter the value of existing solution available in Harmony Memory. This can be done as follows:

$$
\begin{aligned}
& \mathrm{x}_{\text {new }}=\mathrm{x}_{\text {old }}+\operatorname{rand}(0,1) * \text { bw } \\
& \text { Where } \mathrm{x}_{\text {new }}=\text { new solution } \\
& \quad \mathrm{x}_{\text {old }}=\text { old existing solution } \\
& \text { rand }(0,1)=\text { a random value ranging in between } 0 \text { and } 1 .
\end{aligned}
$$

Harmony search a meta heuristic search technique which is generally used to optimize problems whose solution may be analyzed in an n-dimensional space. According to Harmony Search 
Algorithm, each musician plays a musical note to generate the best possible harmony altogether. A new solution is generated for a musical note with the help of harmony factors like HMCR (Harmony Memory Considering Rate), PAR (Pitch Adjustment Rate) and bw (Bandwidth). Each musical note usually possesses its current position. After iteration, the worst harmony is substituted by a new better solution.

The flow chart of Harmony Search is depicted in Figure-1

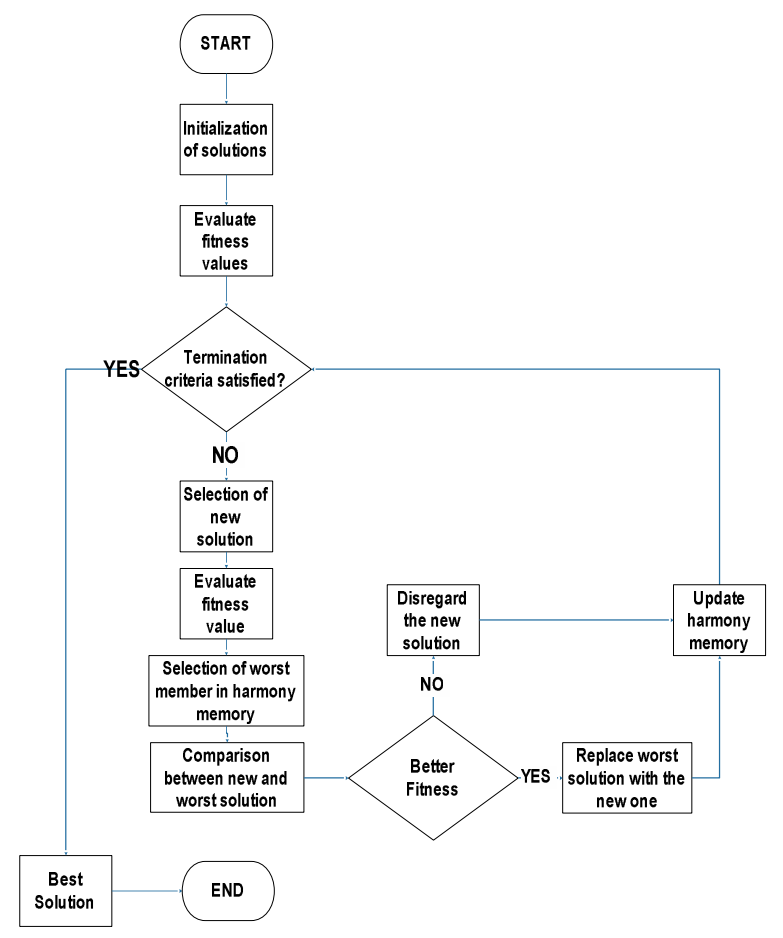

Figure 1: Flow chart of Harmony Search

\subsubsection{PARTICle SWARM OPTIMIZATION}

Particle Swarm Optimization (PSO) is an evolutionary searching method used to optimize problems in an n-dimensional space. It is derived and motivated by the social behavior of birds. In PSO,a potential solution is called particle. In PSO swarm is the combination of different particles and each particle having a candidate solution. Each particle usually posses its current position, current velocity, and its own best position called as pbest. Pbest is the personal best position explored and also incorporates gbest that global best position achieved by all its individuals. It is a simple approach and it is effective across a variety of problem domains.

PSO starts with initialization of particle velocity and current position. In this case the particle is in 2-D space. The fitness value of the particle may be calculated according to function. If the fitness of the particle is better than its previous value, then the position of the particle may be updated to its personal best position. Again if the value of pbest is better than gbest position, the position of global best of the particle is updated. The process may be repeated until termination criteria are met or the optimal solution is found. 
International Journal of Computer Science \& Information Technology (IJCSIT) Vol 8, No 5, October 2016

The ideas behind Particle Swarm Optimization are as follows:

- Every particle has the capability to find the optimal solution.

- Every particle moves with a velocity and position of particle is adjusting through the velocity.

- Pbest is the best position of a particle which gives best result.

- Each particle knows its fitness in its neighborhoods according to the position which gives best fitness functional value.

The flow chart of Particle Swarm Optimization is depicted in Figure 2.

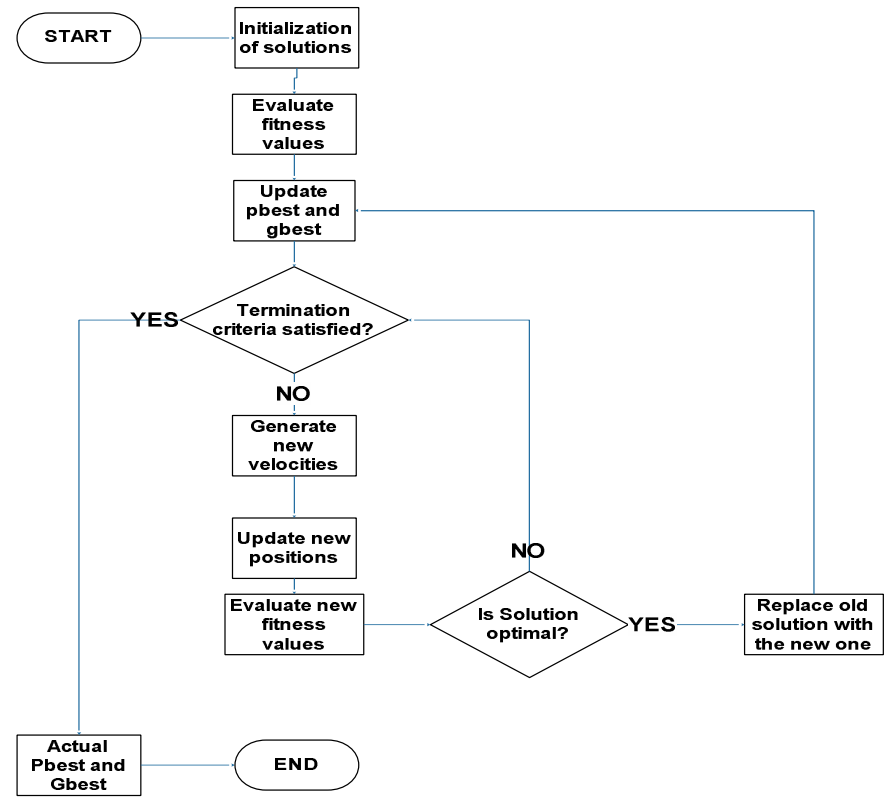

Figure 2: Flow chart of Particle swarm optimization algorithm (PSOA)

The new position of the particle is given by

$\mathrm{x} 1=\mathrm{x}+\mathrm{v} 1$

where $\mathrm{x}=$ candidate solution

$\mathrm{v} 1=$ updated velocity value

$\mathrm{x} 1=$ updated particle position

The velocity for the new solution is given as follows:

$\mathrm{v} 1=\mathrm{w}^{*} \mathrm{v}+\mathrm{c} 1 *($ pbest $-\mathrm{x}) *$ rand $+\mathrm{c} 2 *($ gbest $-\mathrm{x}) *$ rand

where $\mathrm{v} 1=$ new solution

rand $=$ a random number in the range of $[0,1]$

pbest $=$ personal best solution

gbest $=$ global best solution

$\mathrm{c} 1, \mathrm{c} 2$ and $\mathrm{w}$ are the PSO parameters 
International Journal of Computer Science \& Information Technology (IJCSIT) Vol 8, No 5, October 2016

\subsubsection{BeE COLONY Algorithm}

Bee colony algorithm (BCA) is a stochastic method. Behavior of bees is manipulated through this algorithm. Possible set of solutions represent the food source position. The amount of nectar represents the fitness values of all solutions or food source. Generally employed, onlooker and scout bees are available in bee colony algorithm. At first employed bee produces the new solution. Onlooker bee chooses an employed bee to improve its solution. According to the fitness value each candidate solution is done by roulette wheel method through employed bees. Scout Bees are used when the food source is exhausted. The old food source is replaced by new food source randomly through scout bees.

The main steps involved in Bee Colony Algorithm are as follows:

Employed bee initiated the generation of food sources and their fitness functional values are calculated randomly.

According to employed bee new position of food source is generated randomly.

Through onlooker bee selected food sources are improved to produce better results.

Finally the best food source or candidate solution is memorized.

The flow chart of Bee Colony Algorithm is depicted in Figure 3.

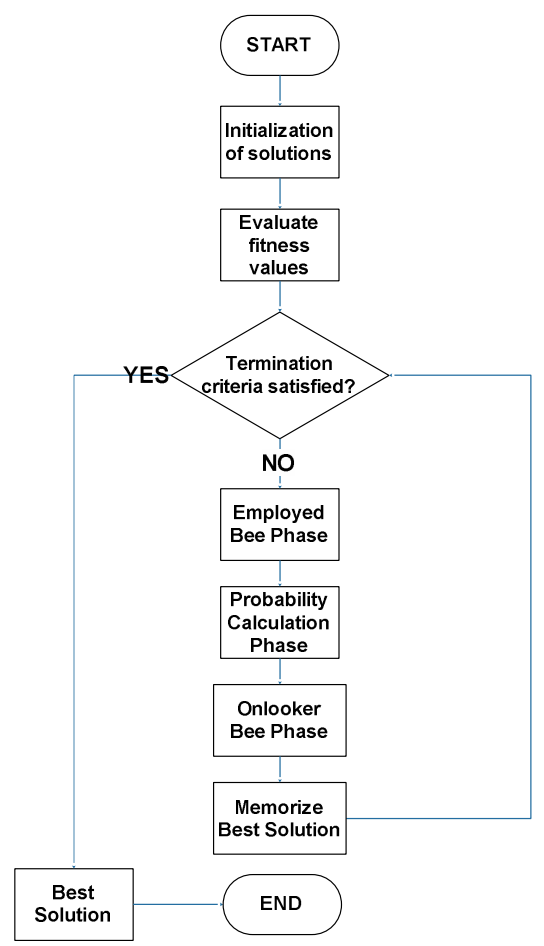

Figure 3: Flow chart of bee colony algorithm

The bee colony algorithm (BCA) having few steps, which are replicated till it ceased. The steps are as follow: 
Step 1.Initially food source population is generated.

Step 2.Evaluate the fitness function values.

Step 3.The current best solution is evaluated.

Step 4.In Employed Bee phase, produce a random solution.

Step 5.Evaluate the fitness function of new solution through employed bee.

Step 6.In Onlooker Bee phase, produce a random solution.

Step 7.According to onlooker bee evaluates the fitness function of new solution.

Step 8. Probability of occurrence of each food source is calculated.

Step 9. Solutions having higher probability factor are improved.

Step 10. Evaluate fitness functional value of the solution and memorize best solution.

Step 11. Continue the process until termination condition reached.

The new solution can be calculated as

$$
c=x(j)+e b f * x(j)
$$

where $x(j)=$ candidate solution at $\mathrm{j}^{\text {th }}$ position

ebf $=$ a random number in the range of $[-1,+1]$

The probability of occurrence for each candidate solution is calculated as follows:

$\operatorname{prob}(\mathrm{j})=\mathrm{fx}(\mathrm{j}) / \mathrm{tfx}$;

Where prob $=$ probability factor

$f x(j)=$ fitness function value

$\mathrm{tfx}=$ total fitness value of all candidate solution

In Onlooker Bee phase, the solution having probability greater than a random value within the range of 0 and 1are selected and there corresponding solutions are improved by using the equation like

$v(j)=x(j)+e b f^{*} x(j j) ;$

where $\mathrm{ebf}=\mathrm{a}$ random number within a range of $[-0.1,+0.1]$

\subsection{Methodology:}

For Mathematical function

$f(x)=1 /(\text { abs }(\text { suc_bal })+\varepsilon)^{2}$

Where $0.1<=\varepsilon<0.9 \quad$ (taking $\varepsilon$-value because overflow condition due to infinity).

Here Successive Amount (suc_amt) is defined as:

suc_bal = net_bal - (wtd_amt - min_bal)

Where net_bal = current account balance

min_bal= minimum bank balance limit

In Harmony Search algorithm(HSA), each solution is initialized with a musical note. The musician will search for optimal solutions by changing the pitch and bandwidth of their musical note. It will keep track of best note in the population and upgrade its solution. Particle swarm optimization algorithm (PSOA) will keep track of the optimal solution which is population-based 
and upgrade its position or location. In Bee Colony Algorithm (BCA) each solution is initialized with the food source. The Employed and Onlooker Bee will search for optimal solution. It will keep track of the population based solution and upgrade its position or location. Optimal solution is used to maximize the mathematical function $\mathrm{f}(\mathrm{x})$ which may be implemented in Harmony search algorithm, PSOA and Bee Colony algorithm using MATLAB-7.0.as shown Table-1. This table primarily focuses on the musician's attempt to generate the best note, particles' movement and movement of employed, Onlooker's Bee to find the best solution in the search space.

Table 1( Fitness Function Value for each sample space or test case)

\begin{tabular}{|l|l|l|l|l|l|l|}
\hline $\begin{array}{l}\text { Iteration } \\
\text { Number }\end{array}$ & $\begin{array}{l}\text { Test } \\
\text { Cases/Test } \\
\text { Data(HSA) }\end{array}$ & $\begin{array}{l}\text { Fitness } \\
\text { Function } \\
\text { Value }\end{array}$ & $\begin{array}{l}\text { Test } \\
\text { Cases/Test } \\
\text { Data(PSO) }\end{array}$ & $\begin{array}{l}\text { Fitness } \\
\text { Function } \\
\text { Value }\end{array}$ & $\begin{array}{l}\text { Test } \\
\text { Cases/Test } \\
\text { Data(BCO) }\end{array}$ & $\begin{array}{l}\text { Fitness } \\
\text { Function } \\
\text { Value }\end{array}$ \\
\hline 1 & 4000 & $5.9488 \mathrm{e}-010$ & 4100 & $5.9779 \mathrm{e}-010$ & 3900 & $5.9198 \mathrm{e}-010$ \\
\hline 10 & 5700 & $6.4746 \mathrm{e}-010$ & 5900 & $6.541 \mathrm{e}-010$ & 4300 & $6.0368 \mathrm{e}-010$ \\
\hline 20 & 7300 & $7.0357 \mathrm{e}-010$ & 7900 & $7.2652 \mathrm{e}-010$ & 5000 & $6.25 \mathrm{e}-010$ \\
\hline 40 & 10800 & $8.5496 \mathrm{e}-010$ & 10000 & $8.1632 \mathrm{e}-010$ & 7100 & $6.9618 \mathrm{e}-010$ \\
\hline 60 & 10800 & $8.5496 \mathrm{e}-010$ & 17200 & $1.2939 \mathrm{e}-009$ & 9700 & $8.025 \mathrm{e}-010$ \\
\hline 80 & 14300 & $1.061 \mathrm{e}-009$ & 21200 & $1.7654 \mathrm{e}-009$ & 13900 & $1.0339 \mathrm{e}-009$ \\
\hline 100 & 16400 & $1.2225 \mathrm{e}-009$ & 25200 & $2.5507 \mathrm{e}-009$ & 17300 & $1.3033 \mathrm{e}-009$ \\
\hline 120 & 18700 & $1.4457 \mathrm{e}-009$ & 29200 & $4.0057 \mathrm{e}-009$ & 27400 & $3.2282 \mathrm{e}-009$ \\
\hline 140 & 20800 & $1.7075 \mathrm{e}-009$ & 33200 & $7.1813 \mathrm{e}-009$ & 43300 & $3.4598 \mathrm{e}-007$ \\
\hline 160 & 24300 & $2.3338 \mathrm{e}-009$ & 37200 & $1.6436 \mathrm{e}-008$ & 44000 & $9.9971 \mathrm{e}-007$ \\
\hline 180 & 26600 & $2.9537 \mathrm{e}-009$ & 41200 & $6.9248 \mathrm{e}-008$ & 44000 & $9.9979 \mathrm{e}-007$ \\
\hline 200 & 29800 & $4.3282 \mathrm{e}-009$ & 44000 & $9.9964 \mathrm{e}-007$ & 44000 & $9.998 \mathrm{e}-007$ \\
\hline 220 & 31000 & $5.1018 \mathrm{e}-009$ & 44000 & $9.9959 \mathrm{e}-007$ & 44000 & $9.998 \mathrm{e}-007$ \\
\hline 240 & 33700 & $7.8313 \mathrm{e}-009$ & 44000 & $9.9962 \mathrm{e}-007$ & 44000 & $9.998 \mathrm{e}-007$ \\
\hline 260 & 35400 & $1.085 \mathrm{e}-008$ & 44000 & $9.9975 \mathrm{e}-007$ & 44000 & $9.998 \mathrm{e}-007$ \\
\hline 280 & 39900 & $3.8445 \mathrm{e}-008$ & 44000 & $9.9969 \mathrm{e}-007$ & 44000 & $9.998 \mathrm{e}-007$ \\
\hline 300 & 43500 & $4.4438 \mathrm{e}-007$ & 44000 & $9.9943 \mathrm{e}-007$ & 44000 & $9.998 \mathrm{e}-007$ \\
\hline 320 & 43800 & $6.943 \mathrm{e}-007$ & 44000 & $9.9969 \mathrm{e}-007$ & 44000 & $9.998 \mathrm{e}-007$ \\
\hline 340 & 44000 & $9.9976 \mathrm{e}-007$ & 44000 & $9.9975 \mathrm{e}-007$ & 44000 & $9.998 \mathrm{e}-007$ \\
\hline 350 & 44000 & $9.9975 \mathrm{e}-007$ & 44000 & $9.9979 \mathrm{e}-007$ & 44000 & $9.998 \mathrm{e}-007$ \\
\hline
\end{tabular}

In this case, 20 numbers of sample test cases are considered. The function value depends upon the parametric values of the input variable. It was found that the solution reaches its optimum value after 198 iterations for particle swarm optimization algorithm (PSOA).After 154 iterations bee colony algorithm (BCA) reaches its optimum value but in case of harmony search algorithm (HSA) takes 325 iterations to get the optimal solution.

\section{Simulation Results}

The proposed approach generates the automated test cases through test cases or test data for Bank ATM by using evolutionary algorithms like Harmony Search, particle swarm optimization and bee colony methods. The figure-4 shows the relation between two variable quantities which are fitness function value range and test data measured along one of a pair of axis represented in table-1. 
International Journal of Computer Science \& Information Technology (IJCSIT) Vol 8, No 5, October 2016

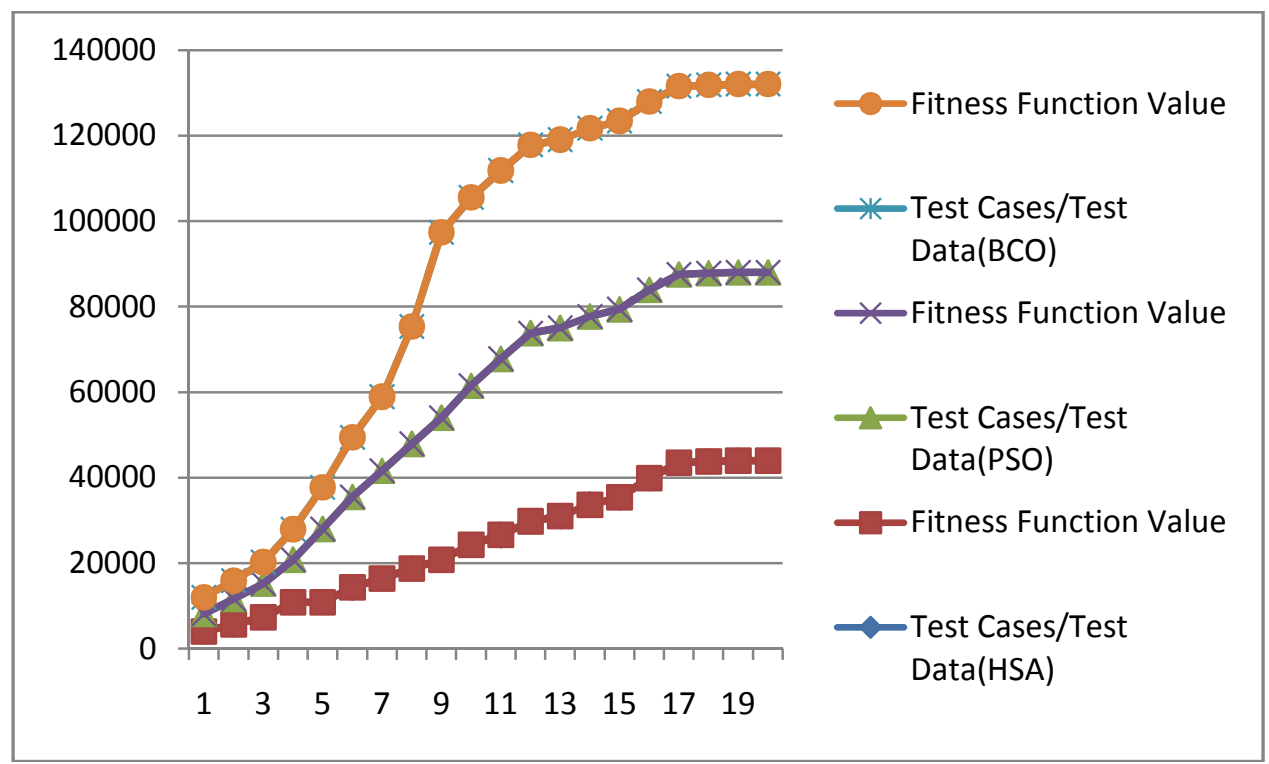

Figure 4: Graphical representation of test data and fitness function value for table-1

The proposed approach generates the test data for Bank ATM's withdrawal operation using Harmony Search Algorithm, PSOA and BCA. Table-2 represents the range of fitness value with different test data and also it gives the individual candidate solution according to the fitness value range in terms of percentage.

Table 2: \%of test data in terms of maximum fitness value

\begin{tabular}{|c|c|c|c|}
\hline FITNESS VALUE RANGE & $\begin{array}{l}\% \text { OF TEST } \\
\text { DATA(HSA) }\end{array}$ & 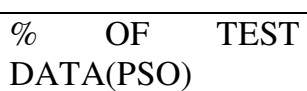 & $\begin{array}{l}\% \text { OF TEST } \\
\text { DATA }(\mathrm{BCO})\end{array}$ \\
\hline $0 \leq \mathrm{f}(\mathrm{x})<0.3$ & 25 & 20 & 10 \\
\hline $0.3 \leq \mathrm{f}(\mathrm{x})<0.7$ & 55 & 20 & 30 \\
\hline $0.7 \leq \mathrm{f}(\mathrm{x})<1.0$ & 20 & 60 & 60 \\
\hline
\end{tabular}

The above table shows that around $60 \%$ test cases or test data are having the higher fitness function $\mathrm{f}(\mathrm{x})$ value and lies in between 0.7 and 1.0 by using bee colony and particle swarm optimization algorithms but in case of harmony search algorithm, only $20 \%$ of test data are available within the higher range of fitness function. By considering all the functional value of fitness function from table 2 bee colony algorithm is having higher fitness value range as compared to particle swarm optimization and harmony search algorithm.Figure-2 shows a pictorial representation of the relation of two variable quantities like percentage of test data and fitness value range. 


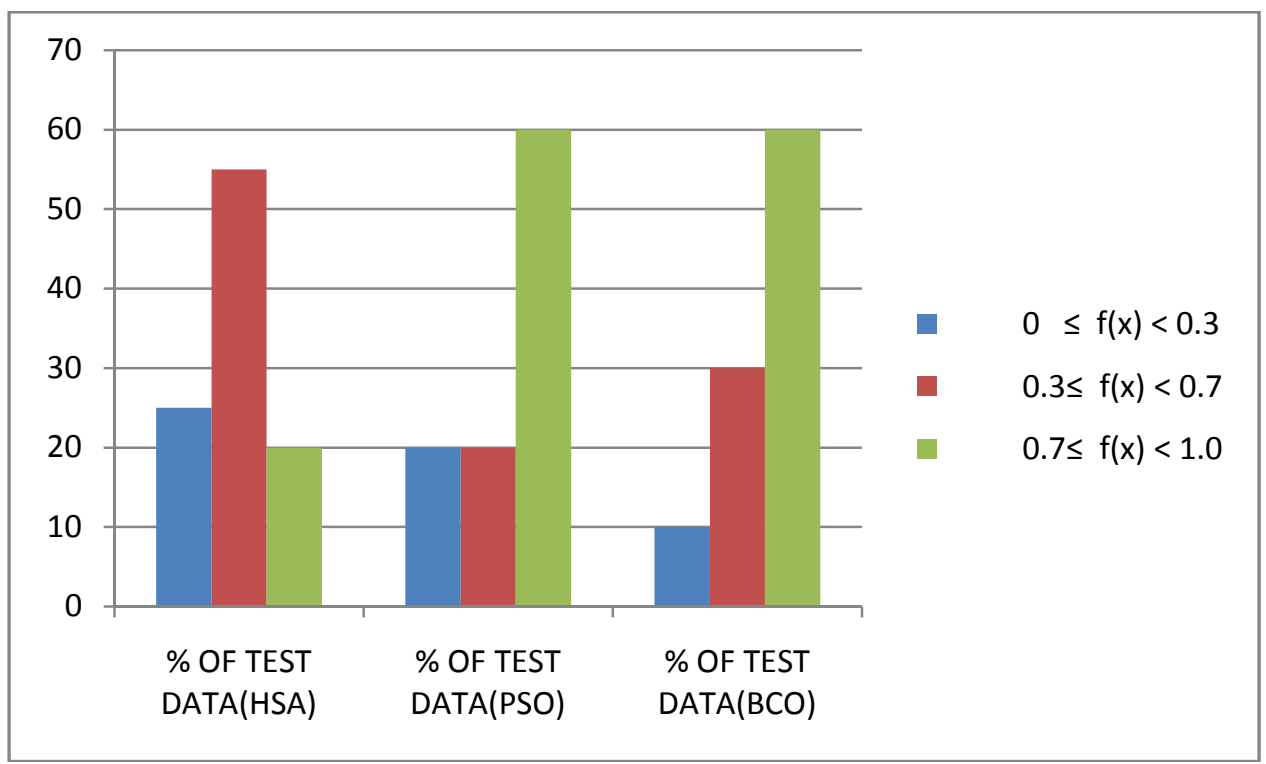

Figure 5: Graphical representation of \% test data and fitness value range for table-2

\section{DisCUSSION AND FUTURE SCOPE}

By considering the mathematical function $\mathrm{f} x=1 /(\mathrm{abs}(\text { net_bal-wd_amt })+\varepsilon)^{2}$, where $\varepsilon$ varies from 0.1 to 0.9 ,this proposed paper generates and optimized the test cases as well as test data automatically through harmony search, particle swarm optimization and bee colony algorithms. According to harmony search with a harmony it has been found that solution of optimality of solution keeps track of best and worst member in the harmony memory and updates its solution accordingly. By considering some sample test cases it has been observed that the functional value depends upon the parametric values of the input variables like Harmony Memory Considering Rate, Pitching Adjusting Rate and the Bandwidth. In case of particle swarm optimization algorithm the particle initialized with current position and velocity it has been found that solution of optimality of particle keeps track of personal best and global best position and updates its position and velocity .In this case the function value depends upon the parametric values of the input variables and the velocity. It has also been seen that the pbest value depends upon the gbest value and pbest is directly proportional to the gbest value along with test cases. In Bee Colony Algorithm (BCA) with the bee initialized with current position it has been found that the optimal solution keeps track of best food source position among a number of food source positions. According to bee colony algorithm the function value depends upon the parametric values of the input variables and food source position.

The future approach to this work could enhance the test case or test data generation for large programs automatically. The different parameters could be added which gives more optimized test cases and also increases the efficiency of Harmony Search (HS), PSOA and BCA techniques. Another perspective area could be the randomly generated test data by using various paths according to the control flow graph (CFG).Test Cases can be generated by using various kinds of hybrid meta heuristic algorithms like BCFA, FABA, BABC etc. The test data generated by using HS algorithm is compared with test data generated by PSOA and BCA and it was found that BCA produces optimal result in very less time and with more accuracy. 
International Journal of Computer Science \& Information Technology (IJCSIT) Vol 8, No 5, October 2016

\section{CONCLUSION}

Evolutionary algorithms like Harmony search (HS), PSOA and bee colony are very important tools for optimization of test cases or test data. It has been diversified the problems in a very effective manner for generating the test data automatically. In this paper, HS, PSOA and BCA have been discussed to generate the optimized test cases by taking an example of withdrawal operation of an ATM machine. This paper also describes the fundamental notions of HSA, PSOA and BCA. It also explains how the random test cases are generated and finding the optimal solution to maximize the problem. This paper will inspire researchers to work on the various evolutionary algorithms by applying in computer science engineering area to generate the effective automated test cases.

\section{REFERENCES}

[1] Arvinder Kaur, Shivangi Goyal "Implementation and Analysis of the Bee Colony Optimization algorithm for Fault based Regression Test Suite Prioritization" International Journal of Computer Applications (0975 - 8887) Volume 41, No.14,March 2012.

[2] Biswal.B.N., Nanda.P., Mohapatra.D.P., 2008 IEEE, "A Novel Approach for Scenario-Based Test Case Generation", International Conference on Information Technology.

[3] B. Korel, "Automated software test generation", IEEE Trans. On Software Engineering 16(8): 870$879,1990$.

[4] Basturk, B., Karaboga, D.: A powerful and efficient algorithm for numerical function optimization: artificial bee colony(ABC) algorithm. In: Proceedings of the IEEE Swarm Intelligence Symposium, pp. 459-471. IEEE, Indianapolis (2006)

[5] C. C. Michael, G. E. McGraw, M. A. Schatz, "Generating software test data by evolution", IEEE Transactions on Software Engineering, vol.27, no.12, pp. 1085-1110, 2001.

[6] Dahiya, S., Chhabra, J., Kumar, S.: Application of Artificial Bee Colony Algorithm to Software Testing. In: Software Engineering Conference (ASWEC), pp. 149-154. IEEE, Auckland (2010)

[7] D. Dervis Karaboga, An Idea Based On Honey Bee Swarm for Numerical Optimization, Technical Report-TR06,Erciyes University, Engineering Faculty, Computer Engineering Department 2005.

[8] Desikan.S. and Ramesh.G,Software testing principles \& practices, Pearson Education,2007.

[9] D. Manjarres, I. Landa-Torres, S. Gil-Lopez et al., (2013)," A survey on applications of the harmony search algorithm”, Engineering Application in Artificial Intelligence. 26(8), pp.1818-1831.

[10] Swagatam Das, Arpan Mukhopadhyay, Anwit Roy, Ajith Abraham, and Bijaya K. Panigrahi,(2011),"Exploratory Power of the Harmony Search Algorithm: Analysis and Improvements for Global Numerical Optimization", IEEE Transactions on Systems, Man, and Cybernetics, Part B: Cybernetics. Vol.41, No.1, pp. 89-106.

[11] Geem, Z.W.,(2009),"Harmony search algorithms for structural design optimization, in Studies in computational intelligence”, Springer Berlin Heidelberg: Berlin, Heidelberg. 23(9), pp. 228-242.

[12] Geem, Z.W.,(2007), "Harmony search algorithm for solving Sudoku", in Proceedings of the 11th international conference, KES 2007 and XVII Italian workshop on neural networks conference on Knowledge-based intelligent information and engineering systems: Part I.,Springer-Verilog: Vietri Sul Mare, Italy.

[13] Geem, Z,(2010),"State-of-the-Art in the Structure of Harmony Search Algorithm", in Recent Advances In Harmony Search Algorithm, Springer Berlin / Heidelberg, pp. 1-10.

[14] H. Sthamer, "The Automatic Generation of Software Test Data Using Genetic Algorithms", PhD thesis, Great Britain, 1996.

[15] J. Kennedy, R. C. Eberhart, "Particle swarm optimization", IEEE International Conference on Neural Networks, Piscataway, NJ., pp.942-1948, 1995.

[16] J. C. Lin, P. L. Yeh, "Automatic test data generation for path testing using Gas", Information Sciences, vol. 131, 2001, pp.47-64. 
International Journal of Computer Science \& Information Technology (IJCSIT) Vol 8, No 5, October 2016

[17] J. Wegener, A. Baresel, H. Sthamer, "Evolutionary test environment for automatic structural testing", Journal of Information and Software Technology, vol. 43, pp. 841-854, 2001.

[18] Kaur Arvinder , Bhatt Divya ,"Hybrid Particle Swarm Optimization for Regression Testing”'International Journal on Computer Science and EngineeringIJCSE),Vol.3,No.5,May 2011.

[19] Mustafa Servet Kiran, Ahmet Babalik "Improved Artificial Bee Colony Algorithm for Continuous Optimization Problems" Journal of Computer and Communications, 2, 108-116, 2014.

[20] R. P. Pargas, M. J. Harrold, and R. R. Peck, "Test Data Generation using Genetic Algorithms", Journal of Software Testing, Verifications, and Reliability, vol. 9, pp. 263-282, 1999.

[21] Glenford J.Myers,(2004), The art of software testing, 2nd ed.: Wiley.

[22] S.Kuppuraj and S.Priya,(2012),"Search-Based Optimization for Test Data Generation Using Genetic Algorithms," in Proc of the 2nd International Conference on Computer Applications, pp.201-205.

[23] Y.Suresh and S.Rath,(2013), "A genetic Algorithm based Approach for Test Data Generation in Basis Path Testing", The International Journal of Soft computing and software Engineering, vol.3, issue.3.

[24] C. C. Michael, G. E. McGraw, M. A. Schatz, "Generating software test data by evolution", IEEE Transactions on Software Engineering, vol.27, no.12, pp. 1085-1110, 2001.

[25] S.K. Swain, D.P. Mohapatra. "Test Case Generation from Behavioral UML Models", International Journal of Computer Applications (IJCA) 6 (2010). 